\title{
PREDICTION OF DIFFERENTIAL CREATININE CLEARANCE IN CHRONICALLY OBSTRUCTED KIDNEYS BY NON-CONTRAST HELICAL COMPUTERIZED TOMOGRAPHY
}

\author{
C.F. NG ${ }^{(1)}$, L.W. CHAN ${ }^{(1)}$, K.T. WONG ${ }^{(2)}$, C.W. CHENG ${ }^{(1)}$, S.C.H. YU ${ }^{(2)}$, W.S. WONG ${ }^{(1)}$ \\ Division of Urology, Department of Surgery ${ }^{(1)}$, and Department of Radiology and Organ Imaging (2), \\ Prince of Wales Hospital, Hong Kong, China
}

\begin{abstract}
Purpose: We investigate the use of non-contrast helical computerized tomography (NCHCT) in the measurement of differential renal parenchymal volume as a surrogate for differential creatinine clearance $(\mathrm{CrCl})$ for unilateral chronically obstructed kidney.

Materials and Methods: Patients with unilateral chronically obstructed kidneys with normal contralateral kidneys were enrolled. Ultrasonography (USG) of the kidneys was first done with the cortical thickness of the site with the most renal substance in the upper pole, mid-kidney, and lower pole of both kidneys were measured, and the mean cortical thickness of each kidney was calculated. NCHCT was subsequently performed for each patient. The CT images were individually reviewed with the area of renal parenchyma measured for each kidney. Then the volume of the slices was summated to give the renal parenchymal volume of both the obstructed and normal kidneys. Finally, a percutaneous nephrostomy $(\mathrm{PCN})$ was inserted to the obstructed kidney, and $\mathrm{CrCl}$ of both the obstructed kidney (PCN urine) and the normal side (voided urine) were measured two 2 after the relief of obstruction.

Results: From March 1999 to February 2001, thirty patients were enrolled into the study. Ninety percent of them had ureteral calculi. The differential $\mathrm{CrCl}$ of the obstructed kidney $(\% \mathrm{CrCl})$ was defined as the percentage of $\mathrm{CrCl}$ of the obstructed kidney as of the total $\mathrm{CrCl}$, measured 2 weeks after relief of obstruction. The differential renal parenchymal volume of the obstructed kidney (\%CTvol) was the percentage of renal parenchymal volume as of the total parenchymal volume. The differential USG cortical thickness of the obstructed kidney (\%USGcort) was the percentage of mean cortical thickness as of the total mean cortical thickness. The Pearson's correlation coefficient (r) between $\% \mathrm{CTvol}$ and $\% \mathrm{CrCl}$ and that between \%USGcort and \% $\mathrm{CrCl}$ were 0.756 and 0.543 respectively. The regression line was $\% \mathrm{CrCl}=(1.00) \times \% \mathrm{CTvol}-14.27$. The $\% \mathrm{CT}$ vol overestimated the differential creatinine clearance by about $14 \%$, but the correlation is good.

Conclusion: The differential renal parenchymal volume measured by NCHCT provided a reasonable prediction of differential creatinine clearance in chronically obstructed kidneys.
\end{abstract}

Key words: kidney; renal function; tomography; x-ray computed; ureteral obstruction

Int Braz J Urol. 2004; 30: 102-108

\section{INTRODUCTION}

Chronic ureteral obstruction commonly causes progressive renal damage. The degree of recovery of renal function of the obstructed kidney af- ter relief of obstruction depends on the severity and duration of obstruction and also the presence of any co-existing infection. In some occasions, the kidney may be so badly damaged that no significant function (the common cutoff point would be less than $10 \%$ 
of the total function) $(1,2)$ can be salvaged. Under these circumstances, the best management may be just a simple nephrectomy rather than any lengthy corrective procedure. In order to make the appropriate management plan, urologists need to know the recovery potential of obstructed kidneys after relief of obstruction. A lot of investigational techniques have been used to assess this potential with various degrees of practicability and accuracy. Commonly used ones include ultrasound measurement of renal cortical thickness, various radioisotope renography and placement of percutaneous nephrostomy (3). In view of its potential accuracy in volumetric measurement and the wide availability of non-contrast helical computerized tomogram (NCHCT), we investigate its use in the measurement of differential renal parenchymal volume in chronically obstructed kidneys, as a surrogate marker for differential creatinine clearance $(\mathrm{CrCl})$.

\section{MATERIALS AND METHODS}

Adult patients with unilateral chronic obstructive uropathy and normal contralateral kidneys, with no overt signs of sepsis (fever, increased leukocytes count, positive urine culture) were enrolled. While the exact duration of renal obstruction could not be easily documented, we defined chronic obstruction as the presence of chronic changes in the radiological appearances, namely dilated pelvicocaliceal system and blunting of calices, with evidence of delayed contrast excretion and drainage in the intravenous urogram. The contralateral kidneys were defined as normal if they were appeared normal radiologically.

Informed consent was obtained for all patients. Ultrasonography (USG) of both the normal and obstructed kidneys was first preformed. The cortical thickness at the site with the thickest renal parenchyma in the upper pole, mid-kidney, and lower pole of both kidneys was measured (Figure-1). Then the mean cortical thickness of each kidney (i.e. the mean of the cortical thickness at the upper pole, mid-kidney and lower pole) was calculated for each kidney. The differential cortical thickness of the obstructed kidney (\%USGcort) was defined as the percentage of the

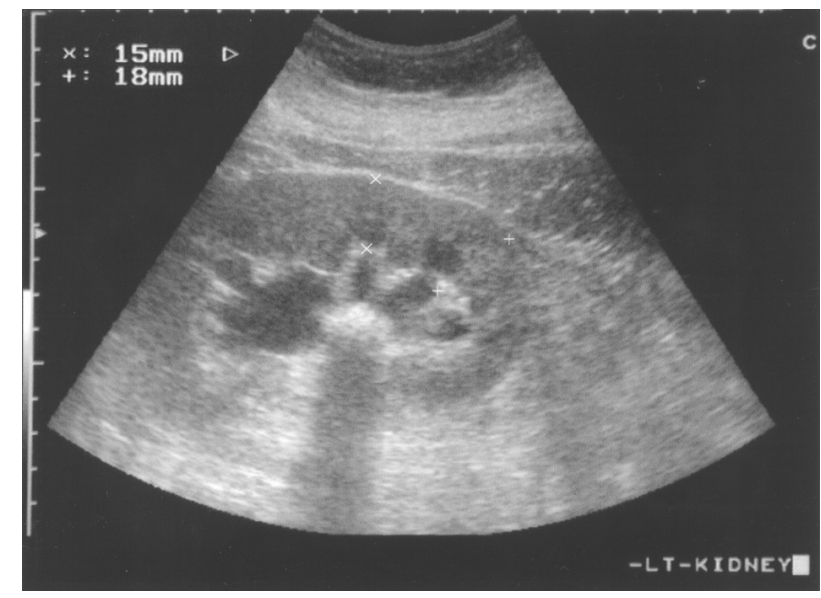

Figure 1-Measuring the renal cortical thickness by ultrasonography.

mean cortical thickness of the obstructed kidney as of the sum of the mean cortical thickness of both kidneys.

NCHCT was then performed using a single slice spiral CT scanner (High Speed Advantage, General Electric, Milwaukee, USA) with $5 \mathrm{~mm}$ collimation, pitch $1.5,120 \mathrm{kV}$ and $210-230 \mathrm{~mA}$. The images were reviewed by a single radiologist who was blinded from the cortical thickness measurement by USG. The cross-sectional areas of renal parenchyma of both kidneys on each slide of images were individually marked with the sinus fat and collecting system excluded (Figure-2). Then the volume of the re-

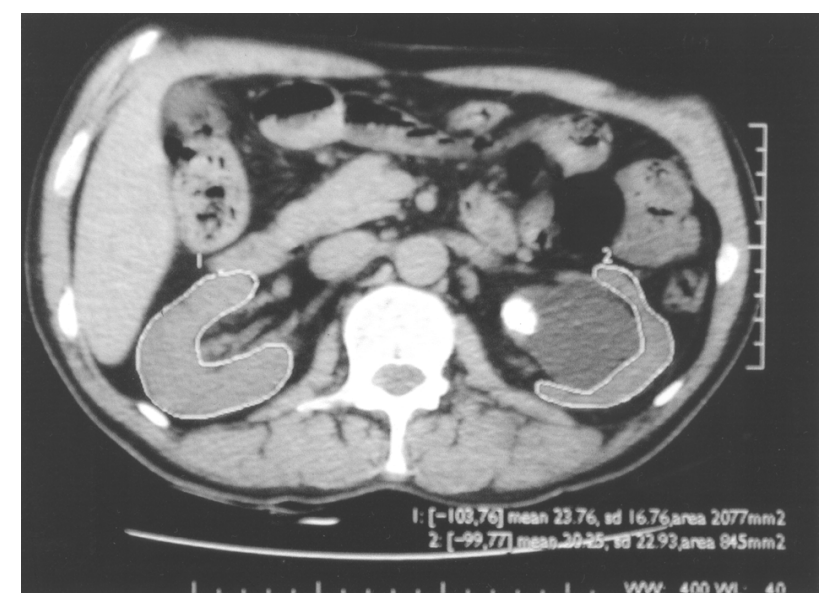

Figure 2 - Measuring the cross-sectional area of renal parenchyma of the kidney on a slide of non-contrast helical computerized tomography images. 
nal parenchyma of each kidney was calculated by summation of the volume of each slide of renal parenchyma (i.e. cross-sectional area times $5 \mathrm{~mm}$ ). The differential renal parenchymal volume of the obstructed kidney (\%CTvol) was defined as the percentage of renal parenchymal volume of the obstructed kidney as of the total renal parenchymal volume of the 2 kidneys.

Finally, an $8 \mathrm{~F}$ percutaneous nephrostomy (PCN) was inserted into the obstructed kidney, and $\mathrm{CrCl}$ of both the obstructed kidney (by collecting the 24-hour PCN urine output) and the normal side (by collecting the 24-hour voided urine) were measured 2 weeks after relief of obstruction. The differential creatinine clearance of the obstructed kidney $(\% \mathrm{CrCl})$ was defined as the percentage of $\mathrm{CrCl}$ of the obstructed kidney as of the total $\mathrm{CrCl}$ of the patient measured 2 weeks after the relief of obstruction. Depending on the differential $\mathrm{CrCl}$ of the obstructed kidney and the clinical condition of the patient, subsequent management was decided accordingly after the study.

\section{RESULTS}

From March 1999 to February 2001, 30 patients (18 males and 12 females) were enrolled into the study. The mean age was 53.8 years (range from 32 to 77 years). Twelve of the obstructed kidneys were the right side and 18 were the left one. Twenty-seven $(90 \%)$ of them suffered from ureteral calculi, 2 suffered from ureteral stricture and 1 from urothelial cancer.

The relationships of \%USGcort, \%CTvol and $\% \mathrm{CrCl}$ were assessed using a linear regression model. The Pearson's correlation coefficient (r) between $\% \mathrm{CT}$ vol and $\% \mathrm{CrCl}$ was 0.756 (Figure-3) and that between \%USGcort and \% $\mathrm{CrCl}$ was weaker, $\mathrm{r}=0.543$ (Figure-4).

The regression line between \%CTvol and $\% \mathrm{CrCl}$ was, $\% \mathrm{CrCl}=(1.00) \times \% \mathrm{CT}$ vol -14.27 .

This implied \%CTvol overestimated the differential renal function at 2 week after relief of obstruction by about $14 \%$, but the correlation is reasonably good.

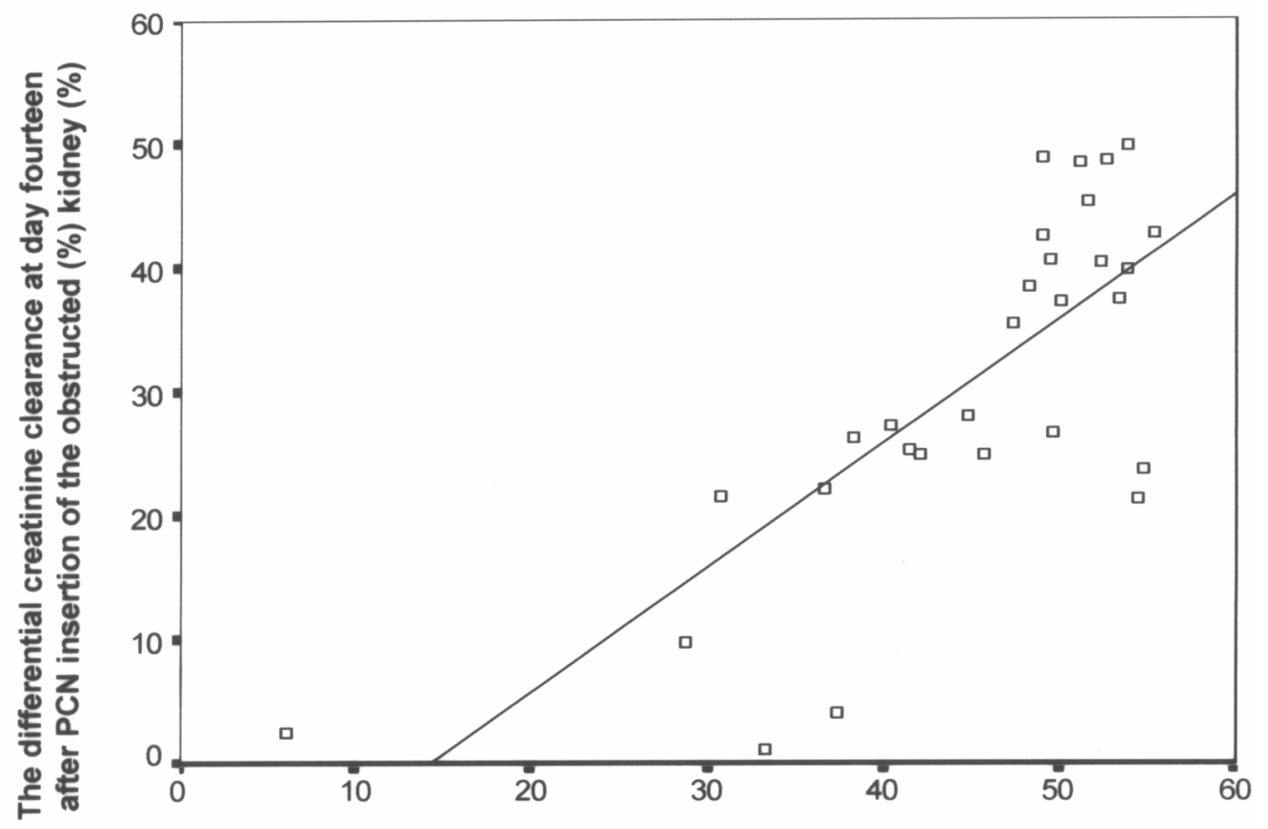

The differential renal parenchymal volume of the obstructed kidney (\%)

Figure 3 - The plotting of the differential renal parenchymal volume of that kidney against the differential creatinine clearance of the obstructed kidney at 14 days post-nephrostomy tube insertion, Pearson's correlation coefficient $=0.756$. 


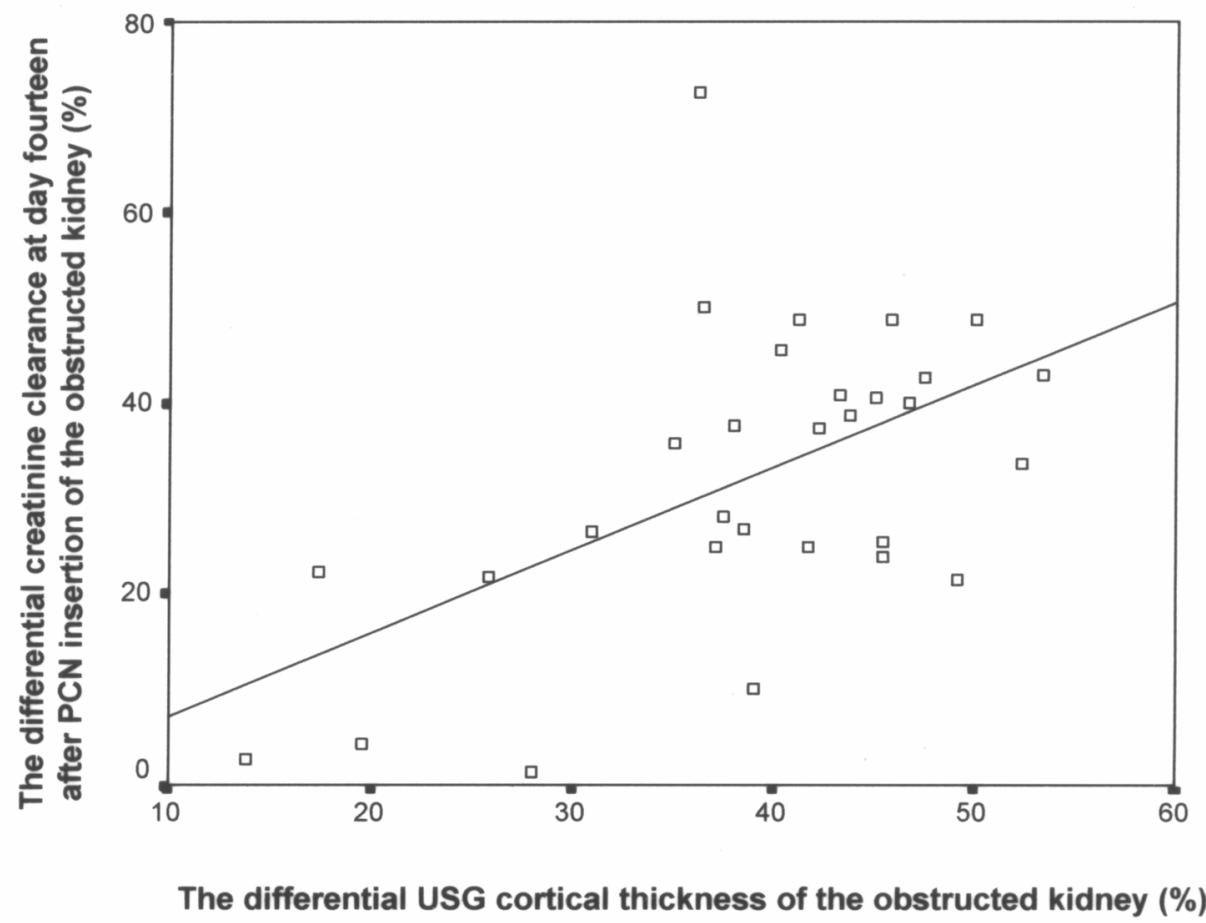

Figure 4- The plotting of the differential ultrasonography cortical thickness of that kidney against the differential creatinine clearance of the obstructed kidney at 14 days post-nephrostomy tube insertion, Pearson's correlation coefficient $=0.543$.

\section{DISCUSSION}

Obstructed kidneys are commonly encountered in urology practice. The most important information required for formulating definitive treatment plan is the cause of the obstruction and the recovery potential of the obstructed kidney. Investigations for the cause of obstruction are quite standardized, including intravenous urogram, computerized tomogram or retrograde pyelogram. If the diagnosis is still uncertain after these imaging procedures, a diagnostic ureteroscopy can usually provide the definitive answer. For the assessment of the recovery potential of the obstructed kidney, various methods are available (3). The commonly used ones are the measurement of cortical thickness by ultrasonography, the various radioisotope renography, and the insertion of $\mathrm{PCN}$ for the measurement of creatinine clearance.

Although ultrasonography is simple, safe, radiation-free and easily available, the lack of standardization in the measurement of the cortical thick- ness and its operator-dependent nature are the main drawbacks. It would not be surprise to have significant inter-observer or even intra-observer variations in the measurement of cortical thickness due to the difference in defining the exact margin of the cortex and renal pelvis, and also the placement and orientation of the measuring markers.

Quantitative measurement of the differential renal function can be provided by radioisotope renography and standardized protocols are available. However, the availability of the procedures and the cost of isotope agents are the limiting factors. Moreover, radioisotope studies are notoriously inaccurate, especially in case of high-grade obstruction, and sometimes PCN insertion is required in order to obtain an accurate assessment (4).

Although being an invasive procedure, the placement of PCN to relieve obstruction and then measure the differential creatinine clearance is still regarded as a simple and reliable method (5). This approach is especially useful when other methods (e.g. 
the radioisotope renography) were not available. Moreover, when the differential function calculated by other techniques was marginal, the placement of PCN to relieve obstruction and then monitoring creatinine clearance of the obstructed kidney for two to three months would help to make the final decision. In our institute, radioisotope renography was not easily accessible and so we frequently required inserting $\mathrm{PCN}$ to assess the differential $\mathrm{CrCl}$ of the obstructed kidneys. The full recovery of renal function for an obstructed kidney requires up to two or three months $(6,7)$. However, patients may find it unacceptable to have the PCN kept in situ for 3 months and there is also significant morbidity associated with prolonged PCN drainage. Therefore, we usually measured the total $\mathrm{CrCl}$ at around 2 weeks after the relief of obstruction. If the calculated differential $\mathrm{CrCl}$ of the obstructed kidney was more than $10 \%$, we would proceed to corrective procedure aiming at relieving the obstruction. On the other hand, if the differential $\mathrm{CrCl}$ was less than $10 \%$, we would keep the PCN for a longer period of time (up to 3 months), to allow time for full recovery in the obstructed kidneys before considering nephrectomy for poorly functioning kidneys. Despite it is a simple and frequently performed procedure, PCN insertion is not without complications, which include bleeding, infection, dislodgement, blockage etc. Therefore, we would like to explore any possible alternative that is easily available in our clinical practice and yet can provide a reasonable prediction of the recoverability of obstructed kidneys, in order to save patients from having $\mathrm{PCN}$ insertion.

NCHCT has already established its role in the diagnosis of acute renal colic in urology practice $(8,9)$. The advantages of NCHCT include its wide availability, high-speed performance and no contrast agents or special preparation required. In view of all these advantages, we would like to explore its potential role in measuring renal parenchymal volume as an indicator to the differential creatinine clearance of kidney.

From our results, differential renal parenchymal volume of the obstructed kidney (\%CTvol) provided a reasonable correlation for the differential $\mathrm{CrCl}$ measured two weeks after the relief of obstruction.
This correlation was better than that between $\%$ USGcort and \% $\mathrm{CrCl}$ (The Pearson's correlation coefficient (r) were 0.756 and 0.543 respectively). The regression line was $\% \mathrm{CrCl}=(1.00) \times \% \mathrm{CTvol}-$ 14.27. Therefore, we could easily calculate the $\% \mathrm{CrCl}$ from this formula, by just deduced 14 from the $\%$ CTvol measured.

In our analysis, we used the differential creatinine clearance at two weeks post-relief of obstruction $(\% \mathrm{CrCl})$ to correlate with the \%CTvol. This might not reflect the differential function of the obstructed kidney after full recovery, i.e. after two to three months (6). However, keeping this limitation in mind, we can still apply the result in clinical practice and improve the patient's care. From our results, for all patients with unilateral chronically obstructed kidneys, NCHCT will be performed and then \%CTvol measured. From the \%CTvol, we can calculate the predicted $\% \mathrm{CrCl}$ of the kidneys. For an obstructed kidney with predicted $\% \mathrm{CrCl}$ less than $10 \%$ (i.e. \%CTvol roughly less than $25 \%$ ), PCN will then be inserted and kept in-situ for up to three months to allow complete recovery of renal function before final decision is made. However, for patients with predicted $\% \mathrm{CrCl}$ greater than $10 \%$, we can proceed directly to definitive management for the obstruction.

We had made an assumption that all the urine from the obstructed kidney was completely drained by the PCN and the voided urine was all from the normal contralateral kidney. However, the intravenous urogram of our patients did not demonstrate complete ureteral obstruction. In fact, except in situation like accidental ligation of ureter, most ureteral obstruction encountered clinically are partial obstruction of various degree. We did not preformed intravenous urogram in our patients after PCN insertion to prove this assumption, because it would increase the radiation exposure of our patient. The basis of this assumption was that after the placement of PCN, the renal pelvis and the ureter proximal to the obstruction would be decompressed and the pressure inside them would decrease. As a result, it is more likely for urine from the obstructed kidney to drain preferentially through the unobstructed PCN than the obstructed ureter. In our opinion, this assumption of complete drainage of urine via the $\mathrm{PCN}$ in an obstructed system was an 
established basis for the use of percutaneous nephrostomy insertion in the measurement of differential function of the obstructed kidney, which had been widely used around the world $(3,5)$. Therefore, we just applied this into our study.

In our study, the measurement of the renal parenchymal volume required the radiologist to mark out the cross-sectional area of renal parenchyma of both kidneys on each slide of images and then summated to give the renal parenchymal volume of each kidney. This was a tedious and time-consuming work. However, with the introduction of workstation system and 3-dimensional volumetric measurement in modern computerized tomogram suite, the renal parenchymal volume can be measured in a faster and perhaps slightly more accurate way (10). This advance in the technology may help to popularize our technique of differential renal function assessment in the future.

\section{CONCLUSION}

The use of non-contrast helical computerized tomography for the measurement of renal parenchymal volume in chronically obstructed kidney demonstrated to have a reasonable correlation to the differential creatinine clearance (as measured by 24 hour urine collection 2 weeks post-relief of obstruction by percutaneous nephrostomy). The incorporation of the ability for functional assessment of kidneys by NCHCT would expand its role in the urology.

\section{REFERENCES}

1. Gulmi FA, Felsen D, Vaughan ED: Pathophysiology of Urinary Tract Obstruction. In: Walsh PC, Retik AB, Vaughan ED, Wein AJ, Kavoussi LR, Novick AC, Partin AW, Peters CA (eds.), Campell's Urology. Philadelphia, Saunders. $8^{\text {th }}$ ed, 2002; pp. 411-62.

2. Whitfield HN: Surgical Management of Renal Stones. In: Whitfield HN, Hendry WF, Kirby RS, Duckett JW (eds.), Textbook of Genitourinary Surgery. London, Blackwell Science. $2^{\text {nd }} e d, 1998 ; 799-812$.

3. Shokeir AA, Provoost AP, Nijman RJM: Recoverability of renal function after relief of chronic partial upper tract obstruction. BJU Int. 1999; 83: 11-7.

4. Van Arsdalen KN, Banner MP, Pollack HM: Radio- graphic imaging and urologic decision making in the management of renal and ureteral calculi. Urol Clin North Amer. 1990; 17: 171-90.

5. Gillenwater JY: Hydronephrosis. In Gillenwater JY, Grayhack JT, Howards SS, Mitchell ME (eds.), Adult and Pediatric Urology. Philadelphia, Lippincott Williams and Wilkins. $4^{\text {th }}$ ed., 2002; pp. 879-905.

6. Jones DA, George NJR, O'Reilly PH, Barnard RJ: The biphasic nature of renal functional recovery following relief of chronic obstructive uropathy. Br J Urol. 1988; 61: 192-7.

7. Cronan JJ: Contemporary concepts in imaging upper tract obstruction. Radio Clin North Amer. 1991; 29: $527-42$.

8. Dalrymple NC, Verga M, Anderson KR, Bove P, Covey AM, Rosenfield AT, et al.: The value of unenhanced helical computerized tomography in the management of acute flank pain. J Urol. 1998; 159: 735-40.

9. Anderson KR, Smith RC: CT for the evaluation of flank pain. J Endourol. 2001; 15: 25-9.

10. Gunn C: Computing. In: Gunn C (ed.), Radiographic Imaging - a practical approach. London, Churchill Livingstone. $3^{\text {rd }}$ edition 2002; 25-35.

Received: December 23, 2003

Accepted after revision: April 15, 2004 


\section{EDITORIAL COMMENTS}

The present paper is a research concerning the use of "non-invasive" technology to assist the urologist/nephrologist in making a clinical decision in management of the patient.

The noncontrast CT scan and calculation of renal volume showed reasonable correlation with creatinine clearance and better correlation than ultrasonography-determined renal volume in the clinical setting where radionuclide image was not an option for these physicians in determining renal function.
The initial noncontrast CT began the decision process of whether the patient then went on to invasive percutaneous nephrostomy for decompression and more exact determination of the renal function for kidney salvage.

In summary, this is an interesting paper with potential impact in management of patients with smaller kidneys and high grade obstruction, since radionuclide studies are notoriously inaccurate in assessing renal function when high-grade obstruction is present.

Dr. William H. Bush, Jr. Director, Genitourinary Radiology University of Washington Medical Center Seattle, Washington, USA

The authors stated that not having the correliterature.

The authors present a useful addition to the

I would like to remember that for measure kidney volume, an alternative to using the summation of individual slices is to do three-dimensional volumetric measurements on a workstation. Where available, this is a quicker and minimally more accurate technique, particularly using helical $\mathrm{CT}$, which has excellent volumetric data. This is important because three-dimensional workstations are becoming available in many institutions.
Dr. Arthur T. Rosenfield Professor of Diagnostic Radiology and Urology Yale University School of Medicine New Haven, Connecticut, USA 